\title{
Node Scheduling based on Grid Optimization for Enhancing Packet Delivery in WSN
}

\author{
Shivani Thakur \\ Department of Electronics and \\ Communication Engineering \\ Maharishi Markandeshwar \\ University, Mullana, India
}

\begin{abstract}
Large number of sensor nodes can be used in WSN (Wireless Sensor Network) and these sensor nodes have limited amount of battery. The algorithm was proposed in pursuance of obtaining maximum lifetime of network. Firstly the area was partitioned into grid and scheduling of nodes based on grid optimization was introduced to enhance the packet delivery between the nodes. Clusters were formed for each grid and all the cluster heads took participation in data transmission. Algorithms with the energy saving and efficiently delivering data to the sink must be developed. However in these algorithms the energy efficiency is declined in the process to deliver data. In this paper, node scheduling strategy is being proposed based on grid optimization. The objective of this paper is to provide an enhanced delivery of data by using grid optimization and enhancing energy efficiency. Evaluation of the proposed algorithm is being conducted by simulations. Evaluated results show that the new proposed algorithm performed better than NSBP [1], comparing in terms of the node death process and network effective lifetime. Node death process is also enhanced in the proposed algorithm over existing one significantly. Leaving the results, that are better than NSBP (A Node Scheduling Based on Partition for WSN), as evaluated through Matlab simulations.
\end{abstract}

\section{Keywords}

Connectivity; grid optimization; partition; weighted sum method; wireless sensor network.

\section{INTRODUCTION}

Sensors in large scale are randomly deployed to observe or monitor the area. As we know large number of nodes is deployed for the real time applications. In case of events like forest fire, surveillance, military purpose, machine health monitoring etc data is being collected. Nodes collect the desired information and pass it from source to sink. As per limitation of energy in case of sensor nodes and difficulty of recharging them which is not a very good option. So, energy efficiency and network effective lifetime have been a matter of great concern in (WSN) wireless sensor network.

To make efficient data delivery and to increase energy efficiency, some techniques must be followed as the redundancy between the nodes must be absolutely avoided for communication. Clustering can be classified as one such approach in which the routing can be performed by clusters, in which every cluster elects a cluster head, then the data is being collected from other nodes and cluster head transmits the data to sink. Node scheduling also plays an important role in increasing the network effective lifetime. There are various states of transition which takes place in scheduling strategies as they may in occur for nodes while some nodes are active only when they are required for data transmission and for rest of the time they are sleeping.
This paper, introduces an algorithm that is based on node scheduling. The key concern behind this protocol is that the data is being collected and is being transmitted to sink based on grid optimization techniques. Thus, this protocol increases the network effective lifetime and node death process that results in energy conservation for the efficient packet delivery. The remainder of this paper is lined up as: Section 2 reviews the NSBP (Node Scheduling Based on Partition for WSN). Section 3 presents the proposed algorithm. Section 4 introduces the simulation results. Section 5 discusses conclusion.

\section{NSBP}

There have been many scheduling schemes for nodes that turn off all the redundant nodes that are idle for extending network lifetime. Many algorithms have transition states of the nodes for their operation based on the desired processes. [1] As in GAF nodes have three states sleeping, discovery, active similarly PEAS also have three states probing, working and sleeping. ASCENT nodes have states four in number as sleep, passive, test and active. In this algorithm coverage and connectivity plays an important role. The GAF and PEAS maintains coverage and connectivity on the grid partition and ASCENT has stable coverage and connectivity. NSBP firstly supports the partitioning of the monitoring area into virtual grid. NSBP defined two modes for working one is a monitoring and other is tracking. The scheduling strategy was introduced in sleep to monitor the area and waking up for tracking events. In the first mode, only one node works in the grid according to scheduling cycle, this extend the lifetime of WSN and also maintains work time. In second mode to get more précised data the numerous nodes were woken up to get more details.

Coverage and connectivity are important and if one node fails to connect other node the connectivity looses and connectivity will be mangled, in order to keep it working it is essential. Coverage is applicable for the overlapping area and so the area is divided into virtual grids. Nodes have probing radius $R_{p}$, based on the grid. It depends on $R_{p}$ and node position. The node when located at the center and completely covers the grid $R \leq \sqrt{2} R_{p}$ is considered as best case. In the worst case node is located at a vertex as $R \leq \sqrt{ } 2 R_{p} / 2$.

$\mathrm{T}_{\text {collect }}$ is the data acquisition cycle, time spent in collecting data is $t_{\text {collect }}$, the idle time is $t_{\text {idle }}$, the scheduling cycle is $\mathrm{T}_{\text {schedule }}(\mathrm{j})$ in $A j, t_{\text {work }}(\mathrm{j})$ is the working time , $\mathrm{t}_{\text {sleep }}(\mathrm{j})$ is the sleep time, therefore

$\mathrm{T}_{\text {collect }}=\mathrm{t}_{\text {collect }}+\mathrm{t}_{\text {idle }}$

$\mathrm{T}_{\text {schedule }}(\mathrm{j})=\mathrm{t}_{\text {work }}(\mathrm{j})+\mathrm{t}_{\text {sleep }}(\mathrm{j})$

In NSBP the mutation of states is based on the round and waking up other nodes is corely based on the queue. The switching of nodes to different states till the last node dies. 
After initialization node goes to sleeping, working and assisting.The results so formed were better than the PEAS algorithm giving network effective lifetime twice as better than it but the results could be more modified and the energy consumption could be efficiently managed through new scheme that is being proposed in the present paper. The network effective lifetime maximization could be achieved through proper grid optimization and considering the worst criteria of node position that is being discussed in the current proposed algorithm.

\section{PROPOSED SCHEME}

This section introduces the proposed algorithm that enhances the network effective lifetime over existing NSBP. This section gives the scheme for enhanced delivery of data by routing that is optimized by grid followed by the grouping into clusters based on the grid distance. The data is transmitted from nodes to the respective cluster heads and these CHs (Cluster Heads) takes part in data transmission and transmits it to the sink. Rest other nodes in a cluster are at sleep, to conserve energy. The cluster heads are being elected among the nodes falling in one cluster and these clusters are formed on the basis of optimized grid formation. The weighted sum method is also used for determining the routing conditions. Various considerations are followed in the proposed algorithm for the network model and sensor nodes.

1) The sink and source are places at opposite ends.

2) The sensor nodes are randomly deployed in the square field.

3) All the nodes are same.

4) Data is collected together and given to the cluster heads as only they take part in transmission.

5) Initial power ranges of the nodes are randomly taken.

\subsection{Grid Partition}

This is the first step of the algorithm in which partitioning is done into equal grids. For the node coverage, grid partition depends upon node position and its transmission range. In the worst case when the two nodes are at the furthest diagonals. The grid distance is calculated based on $R_{t}$ transmission range. If node $\mathrm{P}$ and $\mathrm{Q}$ are at the diagonals and are neighbors than they can communicate only if the distance $d$ is equal or less than $R_{t}$ as shown in Fig 1. The area is in square form.

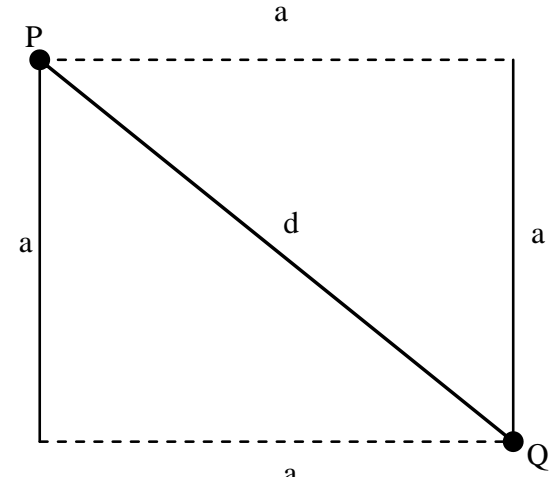

Fig 1: Grid distance

They must satisfy the condition:

$$
\mathrm{d}^{2}=\mathrm{a}^{2}+\mathrm{a}^{2}
$$

So, the grid distance is given as

$$
\begin{aligned}
& d^{2}=\sqrt{ } 2 a^{2} \\
& R_{t}=\sqrt{ } 2 / 2 a
\end{aligned}
$$

This concludes the grid distance according to which now grids can be formed.

\subsection{Clustering}

Once the grids are formed, all the nodes falling in one grid are considered to be in one cluster, as group of nodes forms clusters. Every cluster has its own unique id. The nodes falling in these clusters give all its data to the one of the member of the nodes specifically which is selected as $\mathrm{CH}$ for each round. Each cluster formed has a cluster head. As only cluster heads takes part in routing of data from the source to the sink. It is a crucial phase as each node has a responsibility of data acquisition and transmitting it further to the sink. After every cycle the cluster heads are changed according to the desired conditions. Cluster heads that collects information transmits the data through multi hop in this proposed algorithm, direct or single hoping is not possible as the transmission range is a constraint followed by energy consumption. This approach is responsible uniquely balancing the work between the nodes according to set conditions that effectively result in the formation of a node that is much stronger among others in terms of battery and distance.

\subsection{Cluster Head $(\mathrm{CH})$}

Before routing first work is to elect a cluster head from each grid for the sensor network these cluster heads can vary for every round and the possibility of each node to be a cluster head varies with the conditions applied in the algorithm.

The election of a $\mathrm{CH}$ among the fellow nodes for a cycle is based on battery power and the distance from the sink of each node.

As cluster heads takes part in routing so the energy must be efficiently used by them. Once the cluster heads are formed other nodes goes to sleep, as they do not take part in routing so, as to conserve energy. After one round again the same selection process is being executed for a new cluster head. Usually the battery assigned to each node is being consumed with every action of transmission or sleeping but the one with the highest value and distance from the sink gets the chance to be a cluster head, as the weighted sum method is employed giving more weight age to battery power over distance ,the actual election process is being executed keeping in mind the criteria of energy efficiency. Weighted sum is the method for solving optimization which seeks solution by systematically varying weights among the objective conditions under given constraints this is the way to achieve high performance. The weights are assigned to the battery and distance from the sink, as keeping in mind the equation they must satisfy;

$$
\mathrm{W}_{1} \mathrm{~J}_{1}+\mathrm{W}_{2} \mathrm{~J}_{2}=1
$$

Where, $\quad \mathrm{W}_{1=} \mathrm{W}_{\mathrm{b}}$ is the weight given to the battery.

$$
\mathrm{W}_{2}=\mathrm{W}_{\mathrm{d}} \text { is the weight given to the distance. }
$$$$
\mathrm{J} \text { is the quantity multiplied to the weights }
$$

After the final Selection criteria being set the routing can be performed based on the cluster heads only the cluster heads takes part in routing for one round the heads are different and for another round they may vary and for one round these elected heads will collect data from each cluster and with multi hoping pass it to the sink through the shortest path to the source. For calculating the distance between the nodes the condition must be satisfied:

$$
\mathrm{D}=\left\{\left(\mathrm{X}_{2}-\mathrm{X}_{1}\right)^{2}+\left(\mathrm{Y}_{2}-\mathrm{Y}_{1}\right)^{2}\right\}^{1 / 2}
$$


Neighboring nodes can be calculated for the efficient routing. The $r_{x}$ power and $t_{x}$ power can be subtracted from the nodes after every round. The weighted sum technique gives the option to vary the weights according to desired criteria that can vary effectively.

\section{RESULTS}

The results are evaluated by performing simulations using Matlab. The results so generated are compared with the NSBP. As, the source and sink are placed in the opposite corners of the monitoring area. Data report is being generated after every $10 \mathrm{sec}$ and is transmitted to sink. Factors considered for simulation are given in the Table 1 below.

Table 1. Simulation values

\begin{tabular}{|c|c|}
\hline Factor & Value \\
\hline Area & $50 \times 50 \mathrm{~m}^{2}$ \\
\hline No. of Nodes & 1000 \\
\hline Sleeping Power & $0.03 \mathrm{mw}$ \\
\hline Idle Power & $12 \mathrm{mw}$ \\
\hline Reception Power & $12 \mathrm{mw}$ \\
\hline Transmission Power & $60 \mathrm{mw}$ \\
\hline Initial Energy of nodes & $54-60 \mathrm{~J}$ \\
\hline Transmitting range $\mathrm{R}_{\mathrm{t}}$ & $10 \mathrm{~m}$ \\
\hline Sensing range & $3 \mathrm{~m}$ \\
\hline
\end{tabular}

Simulation result of Node death process:

The grid was built with the grid distance that was approximately $2 \mathrm{R}_{\mathrm{t}} / \sqrt{ } 2$ from (4).

As, Grid distance $=3.5$ and grids of $14 \times 14$ are generated .

1 round $=0.487661 \mathrm{sec}$

The Node death process of PEAS, NSBP and new proposed scheme as shown in Fig 2. Firstly, 1000 nodes are being taken for the process. Vertical direction depicts the Alive number of nodes and horizontal direction gives time slice, where 1 time slice $=1000 \mathrm{sec}$. In case of PEAS nodes completely die about 46th time slice in NSBP it is 85th time slice but in case of proposed algorithm it dies about 155th time slice that is almost double to NSBP. The results are better than NSBP and PEAS of the proposed algorithm.

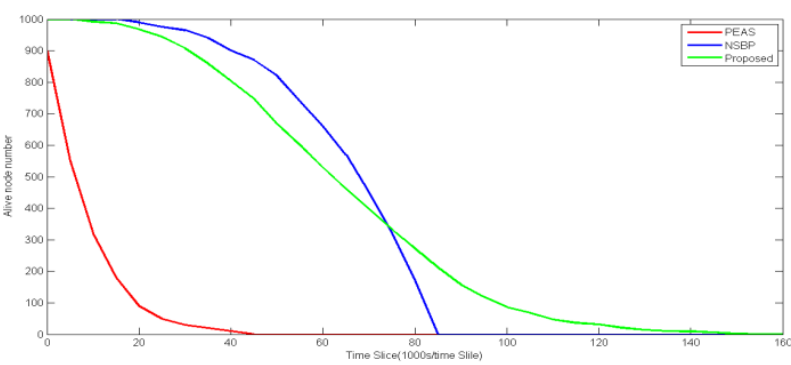

Fig 2: Node death process

Simulation results of Network effective lifetime

Network effective lifetime states that the data efficiently can be transmitted back to sink. This is calculated for the network scale of $200,300,400,500,600,700,800,900$, and 1000 nodes shown in horizontal direction. Proposed algorithm performed better over NSBP as shown in Fig 3.
The values obtained in case of NSBP are much lesser than what is being obtained by the proposed algorithm. The performance of Network effective lifetime shows that the network will live out more and can be lengthened.

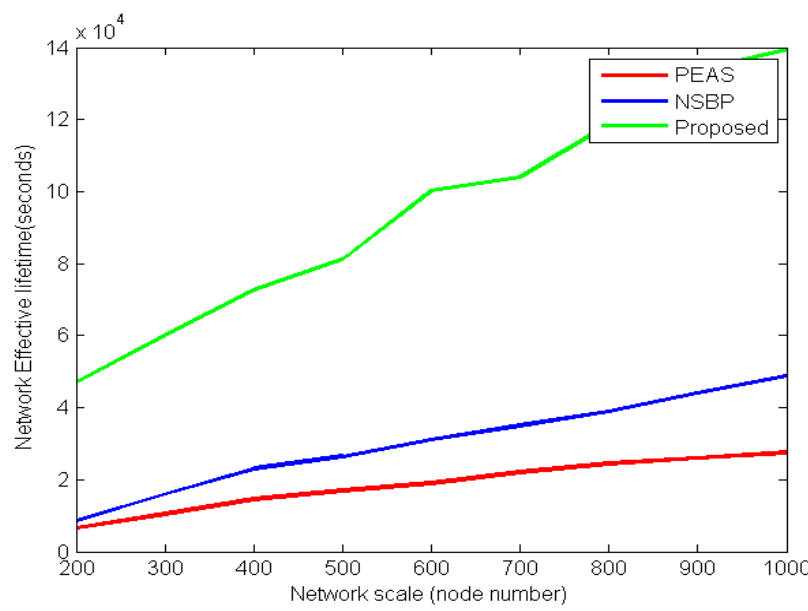

Fig 3: Network Effective Lifetime vs. Network Scale

For comparison between the effective lifetimes, Nodes death time and effective lifetime can be compared taking scale of 1000 nodes. Fig 4 shows that the effective lifetime of 1, 2, 3 as shown for PEAS, NSBP and proposed algorithm respectively which shows that proposed algorithm is better than all of them.

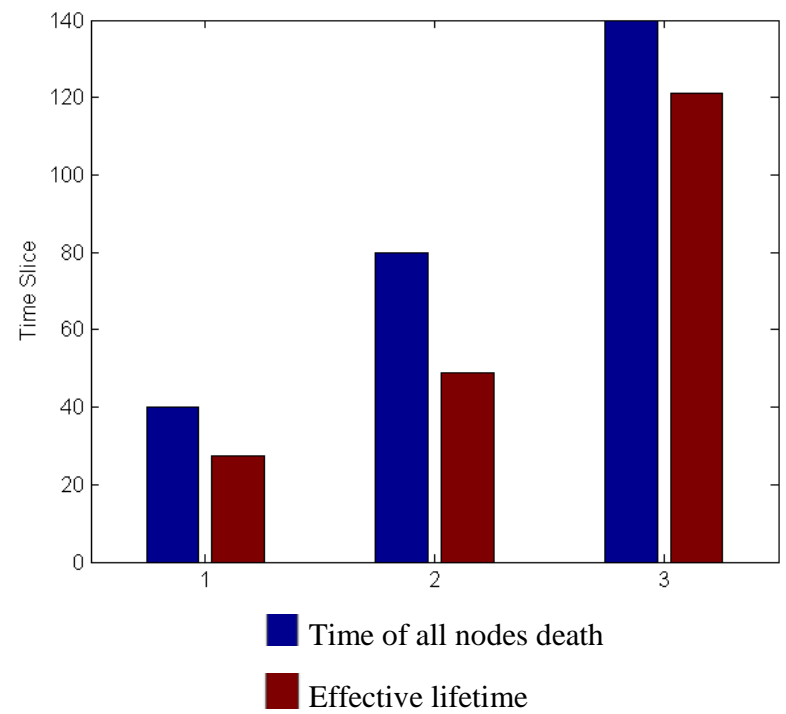

Fig 4: Comparison between effective lifetimes

\section{CONCLUSION}

Node scheduling based on grid optimization that efficiently delivered packets to the sink was introduced. The nodes make use of multi hop for the transmission. Taking into account of grid partition that enables efficient conservation of power by switching other nodes into sleep mode by assigning the cluster heads and allowing them to take part in transmission for every single round. Performance of the node scheduling algorithm based on grid optimization showed improved results over the discussed algorithm and which gives improved effective lifetime. This algorithm can be applied for its simplicity and energy conservation for the applications that requires enhanced network effective lifetime with energy conservation 
for diligent data delivery .The network will survive for a longer time.

\section{REFERENCES}

[1] Yimei Kang, Yang Han, "A Node Scheduling Based on Partition for WSN (NSBP)," in Wireless Telecommunications Symposium (WTS) IEEE, pp. 1-6, April 18-20, 2012.

[2] Maciej Nikodem and Bartosz Wojciechowski, "Upper Bounds on Network Lifetime For Clustered Wireless Sensor Networks," in 4th IFIP International Conference on New Technologies, Mobility and Security (NTMS), pp. 1-6, Feb 7-10, 2011.

[3] Dahlila P. Dahnil, Yaswant P. Singh, Chin Kuan Ho, "Energy-Efficient Cluster Formation in Heterogeneous Wireless Sensor Networks: A Comparative Study," in 2011 30th International Conference on Advanced Communication Technology (ICACT), pp. 746-751, February 13-16, 2011.

[4] Chi-Tsun Cheng, Chi K. Tse, and Francis C. M. Lau, "A Clustering Algorithm for Wireless Sensor Networks Based on Social Insect Colonies," IEEE Sensors Journal, Vol. 11, Issue: 3, pp. 711-721, March 2011.

[5] Chi-Tsun Cheng, Chi K. Tse, Francis C. M. Lau, "A Delay-Aware Data Collection Network Structure for Wireless Sensor Networks," IEEE Sensor Journal, Vol 11, Issue: 3, pp. 699-710, March 2011.

[6] Chutima Prommak , Sujitra Modhirun , "Optimal Wireless Sensor Network Design for Efficient Energy Utilization," in IEEE Workshops of International Conference on Advanced Information Networking and Applications (WAINA) , pp. 814-819 , March 22-25, 2011.

[7] Vivek Katiyar, Narottam Chand , Surender Soni, "Improving Lifetime of Large-scale Wireless Sensor
Networks through Heterogeneity," in International Conference on Emerging Trends in Electrical and Computer Technology (ICETECT), pp. 1032-1036 , March 23-24, 2011.

[8] Hamed Yousefi, Mohammad Hossein Yeganeh, Ali Movaghar, "Long Lifetime Routing in Unreliable Wireless Sensor Networks," in International Conference on Networking, Sensing and Control, (ICNSC), pp. 457 462, April 11-13, 2011.

[9] Jing-hui Zhong, Jun Zhang , "Energy-Efficient Local Wake-up Scheduling in Wireless Sensor Networks," in IEEE Congress on Evolutionary Computation (CEC) , pp. 2280-2284, June 5-8, 2011.

[10] Sounak Paul, Naveen Kumar Sao, "An Energy Efficient Hybrid Node Scheduling Scheme in Cluster Based Wireless Sensor Networks," in Proceedings of the World Congress on Engineering WCE 2011, Vol II, July 6 - 8, 2011.

[11] Moslem Noori, Masoud Ardakani, "Lifetime Analysis of Random Event-Driven Clustered Wireless Sensor Networks," in IEEE Transactions on mobile computing, Vol 10, Issue: 10, pp. 1448-1458, October 2011.

[12] Hamid Rafiei Karkvandi, Efraim Pecht, Orly YadidPecht ,"Effective Lifetime-Aware Routing in Wireless Sensor Networks," in IEEE Sensor Journal , Vol 11, Issue: 12, pp. 3359-3367, December 2011.

[13] Hwa Young Lim, Sung Soo Kim, Hyun Jun Yeo, Seung Woon Kim, and Kwang Seon Ahn, "Maximum Energy Routing Protocol based on Strong Head in Wireless Sensor Networks," in IEEE sixth International Conference on Advanced Language Processing and Web Information Technology, ALPIT 2007, pp. 414-419, Aug 22-24, 2007. 